\title{
Feature-index-based similar shape retrieval
}

\author{
R. Mehrotra \\ Departement of Mathematics and Computer Science \\ University of Missouri-St. Louis \\ 8001 Natural Bridge Road \\ St. Louis, MO 63121, USA rajiv@arch.umsl.edu \\ J. Gary \\ Department of Computer Science \\ University of Kentucky \\ Lexington, KY 40506, USA jgary@ms.uky.edu
}

\begin{abstract}
Efficient retrieval of images containing matching (or similar) shapes is an important problem in image database management. A few shape similarity-based image retrieval techniques have been reported in the literature. Most of these techniques are designed to deal only with images of rigid objects and cannot easily handle shape similarity-based retrieval of images of articulated objects (i.e., objects with one or more joints with movable components). Some of these techniques are incapable of handling images of overlapping and touching objects. In this paper, a new feature-index-based shape similarity-based image retrieval technique, which is capable of handling images of rigid as well as articulated objects, is proposed. The proposed technique can also retrieve images of overlapping and touching objects that satisfy the query constraints. In this approach structural components of a shape called features are represented as points in a multidimensional space. Any multidimensional point access index structure can be used to organize shape features. Given a query shape or image, a query shape feature is selected and similar (or matching) shape features belonging to images in the database are found by searching the feature index structure. If the query shape and a stored shape containing any of the retrieved matching features are found to be similar (or satisfy similarity constraints), the stored shape is included in the response to the query.
\end{abstract}

\section{Keywords}

image database, content-based retrieval, feature indexing, shape similarity retrieval 


\section{INTRODUCTION}

With recent advances in computer technologies, numerous new application areas requiring management of pictorial data have evolved. Efficient management of pictorial data is required in areas including meteorology, medical, space exploration, manufacturing, entertainment, education, and defense. In these, and other, application areas large and constantly growing repositories of images are available. But such image repositories and of little use due to the lack of techniques for image retrieval based on their content or semantics. In recent years, there has be an ever increasing interest in developing content-based image retrieval techniques.

Similarity of two images can be defined by general image characteristics such as intensity, color, texture, etc. (Faloutsos, 1993, Barber, 1993), in terms of spatial relationships among image objects (Chang, 1987), or in terms of the shapes present in the images. This paper is concerned with the problem of shape similarity based retrieval. In shape similarity based image retrieval, stored images that satisfy the specified shape similarity constraints with respect to a query image are required to be retrieved from the database. In simple terms, shape similarity-based image retrieval can be stated as follows:

Given a shape similarity measure, retrieve (or select) all images containing shapes similar to one or more shapes present in the query image.

The focus of this paper is on image databases of two-dimensional (2D) (i.e., flat or almost flat) objects/shapes ${ }^{1}$. Two types of $2 \mathrm{D}$ objects are usually found:

1. Rigid objects - These objects have a single rigid component (or link).

2. Articulated objects - These objects are made up of two or more rigid components called links joined by movable joints (rotating or sliding). Examples of articulated objects include a pair of scissors, a pair of pliers, etc.

An ideal shape similarity-based retrieval method must be general enough to handle images of articulated as well as rigid objects. Furthermore, in an uncontrolled environment, objects may overlap and touch each other. Such cases result in complex shapes (or images) caused by the occlusion or overlapping of the object. An ideal shape retrieval techniques must be flexible enough to handle images with overlapping or touching objects (referred to as complex images) as well as images of isolated shapes (referred to as simple images.

Existing shape similarity-based image retrieval techniques can be broadly classified into two classes. Techniques belonging to one of these classes rely on specially designed index structures for shape retrieval whereas the techniques in the other class utilize existing point access methods or spatial access methods.

Grosky and Mehrotra (Grosky, 1989, 1990, 1992) proposed a technique which represents

\footnotetext{
${ }^{1}$ The terms object and shape are used interchangeably in this paper.
} 
a shape in terms of local structural features of its boundary. They proposed a specialized index structure based on the $m$-way search tree to organize boundary components of shapes stored in the image database. This technique can handle images of occluded and touching objects, but the index structure is very complex.

Jagadish's technique (Jagadish, 1991) represents a shape in terms of the properties of a fixed number of largest size rectangles from the set of rectangles that cover the shape. A shape is represented as a point in a multidimensional space and therefore any multidimensional point access method (PAM) (Nievergelt, 1984, Robinson, 1981), Seeger, 1987) can be used for indexing. The shape representation utilized in this techniques is suitable only for isolated shapes and therefore it cannot handle images with occluded or touching objects.

Recently, Mehrotra and Gary (Mehrotra, 1993, Gary, 1993) proposed an approach which combines the strength of the above two techniques while overcoming some of their weaknesses. Their approach represents an object in terms of its local structural boundary features. Each structural feature is represented as a point in a multidimensional space. Thus, any existing multidimensional PAM can be used to build a feature index to efficiently organize the structural features of all the stored shapes or images. This approach is capable of dealing with images of overlapping and touching objects.

In Mehrotra-Grosky's and Mehrotra-Gary's techniques, for a given a query image, a query feature (i.e., a structural boundary component of the query shape) is selected from the query image and the matching features are found by searching the index structure. Associated with each feature in the index is a list of (parent-shape, feature-location) pairs, which tells where and in which shape that feature occurs. The lists associated with the matching features are used to retrieve the shapes that satisfy the specified similarity criteria.

A very few computer vision researchers have considered the problem of recognition of articulated objects. Beinglass and Wolfson (1991) describe a generalized Hough transform approach. Their approach is similar to the one presented here, but uses a "voting" procedure in generation of hypotheses. Lowe (1991) describes the use of least squares model fitting for parameterized models to images. This approach is quite flexible and may have application in pose estimation once hypotheses have been identified, but is too time consuming for an interactive query session in a large database.

Most existing approaches of similar shape retrieval lack the capability of dealing with articulated objects in a simple way in the context of a large database. One possible way to handle articulated shape retrieval using these methods is to automatically generate a sufficiently large number of shapes corresponding to different relative positions of the components of a query shape. Then for each generated shape, similar shapes can be retrieved. Obviously, this approach is impractical due to its very high time requirement. An alternative approach, which is adopted in this work, is to employ a general transformation invariant shape representation method which can represent both the rigid as well the articulated object in the same way. In this paper, a general and flexible shape similarity-based retrieval approach is proposed. This approach represents an articulated 
shape in terms of its rigid components and articulation points. Each rigid component is represented in terms of its boundary components which are in turn represented as points in a multidimensional space. The proposed technique is described in the following sections. Some experimental results are also presented to demonstrate its performance.

\section{FEATURE BASED SIMILARITY RETRIEVAL}

Three primary issues to be considered in designing a shape similarity based image retrieval system are shape representation, similarity measure, and index design. The symbolic shape representation should be flexible enough to allow retrieval of isolated images and images with overlapping or touching parts. The representation should also be general enough to handle rigid and articulated shapes. The similarity measure should be consistent with human interpretation and efficiently computed. It is also advantageous for the similarity measure to be a metric. Together, the representation and similarity measure should lend themselves to a efficient range search method to facilitate retrieval of similar or matching shapes. A specially designed index could be constructed, or existing indexing techniques could be used.

\subsection{Iconic index construction}

Each shape to be stored in the database is first processed to obtain the shape boundary. Points on the boundary with maximal local curvature are chosen as interest points. The details of this process are not addressed in this paper. A variety of techniques have been developed for the robust identification of interest points (Tsang, 1994, Wuescher, 1991). The object is thus represented as an ordered sequence of interest points. We define a feature to be a collection of a few adjacent interest points. In other words, a feature represents a boundary segment and the shape is a set of boundary segments. We have experimented with 4, 5, and 6 interest point per feature. Each feature is encoded to make it scale, rotation, and translation invariant, and entered into the index structure. The steps involved in index construction are illustrated in figure 1.

\subsubsection{Feature encoding}

Given a set of $n$ interest points, a pair is chosen to form a basis vector. A coordinate system is defined with this vector as a unit vector along the $x$ axis. All other interest points in the feature are transformed to this coordinate system. Figure 1 illustrates this transformation. This form of encoding is essentially arbitrary; any pair of points could form the basis vector, or indeed all pairs could be used. In our experiments, we select only adjacent interest points for feature encoding. The vector $F=\left(x_{1}, y_{1}, \ldots, x_{n}, y_{n}\right)$ where $\left(x_{i}, y_{i}\right)$ is the normalized coordinate of the $i^{\text {th }}$ interest point in that feature, is used as a key in the index structure. Each feature is characterized by the transformation used to encode the interest points. This is represented as an attribute vector $P=\left(S, T_{x}, T_{y}, A\right)$ where $S$ is the scale, or length of the basis vector, $T_{x}$ and $T_{y}$ are the translation parameters, or location of the tail of the basis vector, and $A$ is the angle the basis vector forms with the $x$ axis. Thus a feature of $n$ interest points is represented as a point in $2 n+4$ dimensional 


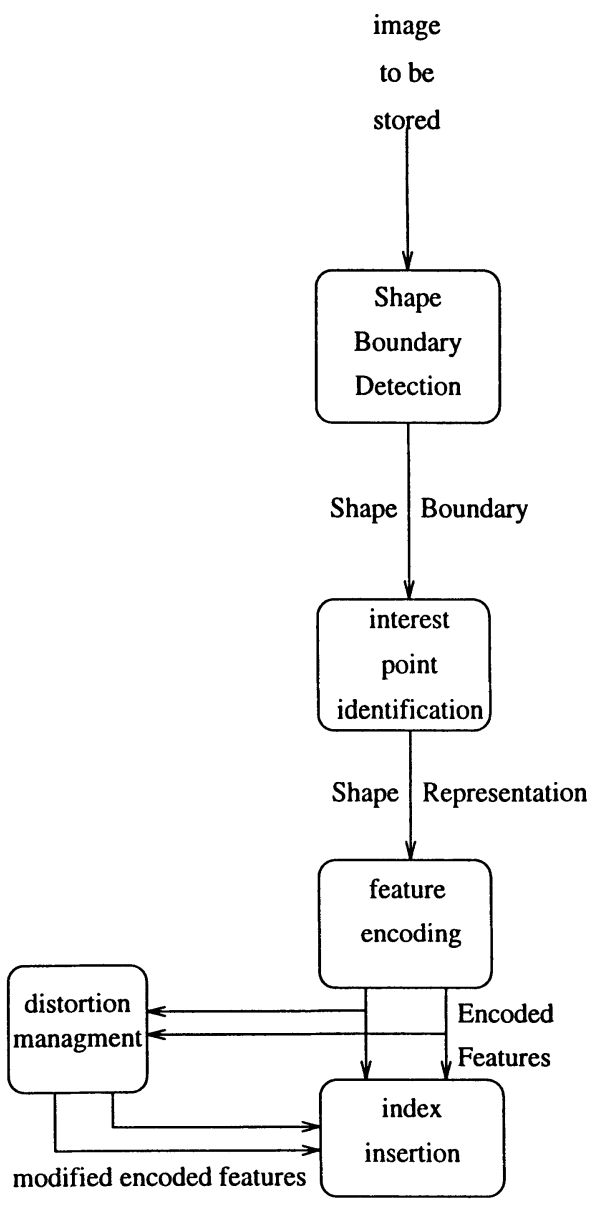

Figure 1: Index Construction 

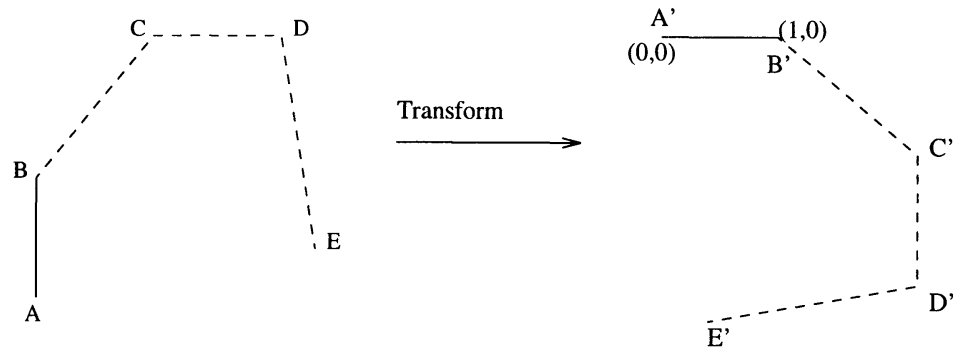

Figure 2: Feature Encoding Example

space. The Euclidean distance between two points is used as a measure of the similarity of the corresponding boundary features.

\subsubsection{Shape representation}

A rigid shape with $m$ interest points is represented by a set of boundary features $\left(F_{1}, \ldots, F_{m}\right)$, its object identifier, and its model type (rigid). A feature is generated for each set of $n$ adjacent interest points. For example, if a shape was represented by interest points $\left(I_{1}, \ldots, I_{m}\right)$, where $I_{i}=\left(x_{i}, y_{i}\right)$ and feature size was chosen to be four, then $F_{1}=\left(x_{1}, y_{1}, x_{2}, y_{2}, x_{3}, y_{3}, x_{4}, y_{4}\right), F_{2}=\left(x_{2}, y_{2}, x_{3}, y_{3}, x_{4}, y_{4}, x_{5}, y_{5}\right), \ldots, F_{m}=$ $\left(x_{m}, y_{m}, x_{1}, y_{1}, x_{2}, y_{2}, x_{3}, y_{3}\right)$. Each boundary feature is defined by its transformation parameter set, $P$. An articulated object with $n$ components is represented by a collection of its rigid components $\left(C_{i}, \ldots, C_{n}\right)$, its object id, and its model type (articulated). The representation of a rigid component of an articulated shape is similar to that of a rigid object, but is augmented with a list of articulation point locations (in the normalized coordinate system) and the identifiers of components associated with those articulation points. A rigid component $C$ with $m$ interest points of an articulated object is represented by a set of boundary features $\left(F_{1}, \ldots, F_{m}\right)$, a set of articulation points $\left(\left(x_{1}, y_{1}\right), \ldots,\left(x_{n}, y_{n}\right)\right)$ where $n$ is the number of articulation points on the component, and identifiers for the components $\left(i d_{1}, \ldots, i d_{n}\right)$, that share each articulation point. 


\subsubsection{Index structure}

The encoded vectors representing the boundary features are used to form a feature index for the shape database. The similarity between two features is defined as the Euclidean distance between the two vectors. Given this structure and similarity measure, our index can take the form of any multi-dimensional point access method (PAM). Several such data structures have been designed to be used with large databases on external storage and to support range searches. These structures are generally hierarchical. The encoded feature vectors serve as the key values and associated with each leaf of the index structure is a list where each node stores information about where and in what shape this feature appears. Specifically, for features of rigid shapes, the node consists of a (transformation parameters, object identifier) tuple. Articulated shape features have an entry for each articulation point in that component in the form of a (transformation parameters, object identifier, articulation point location, sibling component identifiers) tuple, where sibling components are the ones that share the articulation points. The list associated with a feature is used to generate a set of hypotheses regarding shapes in the image database that may satisfy shape similarity constraints with respect to a given query shape. In principal, the transformation parameters could be made part of the feature vector and used in the index search, but doing so would increase the dimension of the search space and reduce the efficiency of the search if the query is intended to ignore their values. Our focus is on finding features of similar shape independent of transformation parameters to hypothesize a set of possibly similar objects (objects that contain similar features). Hypothesized shapes may be rejected if they fail to meet similarity constraints or specified restrictions on transformation parameters.

\subsection{Query processing}

Processing shape similarity queries involves three phases, query feature selection, hypothesis generation, and hypothesis verification. The system selects a feature from the query shape using criteria designed to maximize the chances for a successful search. The index is then searched for features similar in shape to the selected feature. The tolerance on similarity is specified in the query and since the similarity measure is Euclidean distance, a range search is performed on the index. For each feature found to be similar in the search, each object where the feature is present is added to the set of hypotheses. The set of hypotheses contains shapes which have some feature similar in shape to the query feature. The verification phase then attempts to reject those shapes that are not globally similar in shape to the query shape. We describe the details of these phases below and illustrate the steps involved in figure 3.

\subsubsection{Query feature selection}

There are a few issues to be considered in selection of a feature from the query shape for searching. We wish to minimize the amount of time spent searching, so we should attempt to choose a feature that has a good chance of generating all valid hypotheses. 


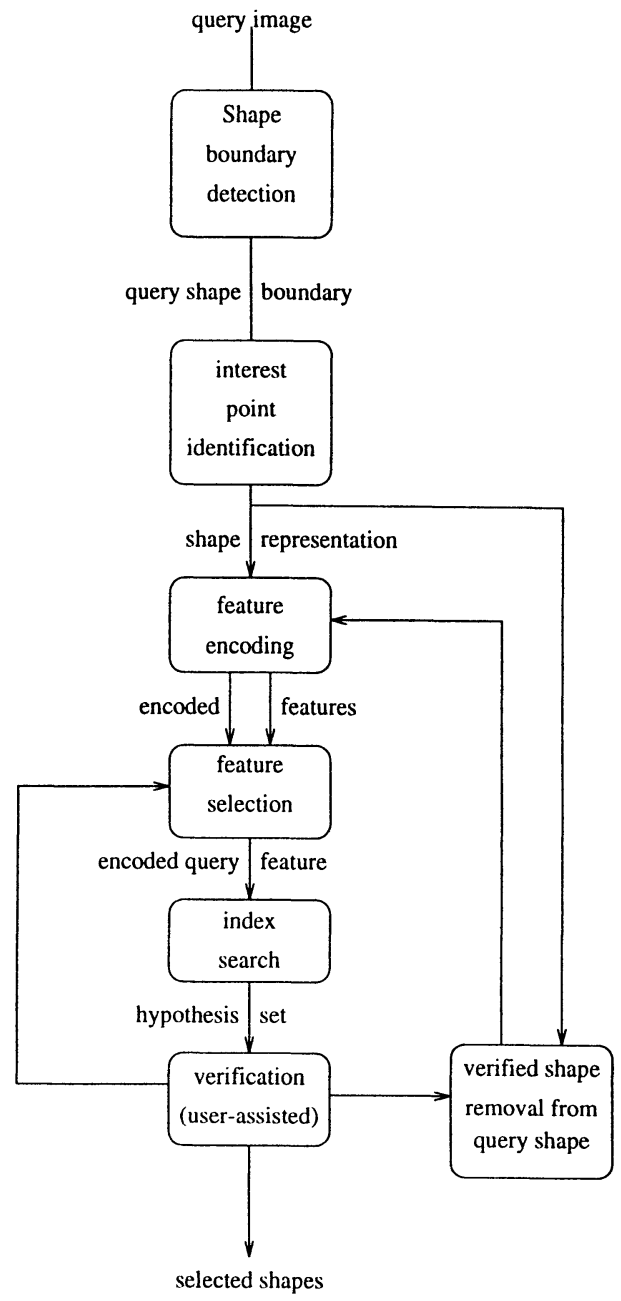

Figure 3: Query Processing 
We also prefer features that are less sensitive to noise and distortion in the input image.

It should be noted that, for our feature representation, the intersection of articulated components (or occluding objects in general) will most likely produce an interest point, since the overlap should produce a well defined corner point. This interest point will cause a concave angle in the boundary representation, except in the unlikely case of two corners of the overlapping objects coinciding. It seems reasonable to choose only interest points that do not form concave angles in the boundary. Unfortunately, it is not always possible to find $n$ adjacent interest points with no concave angles.

The problem of distortion in an input image is partially accounted for by adjusting the tolerance on similarity during the search. It should also be noted that small basis vectors will magnify errors more than large basis vectors. Distortion in an input image could also produce false interest points, but such points should form shallow angles in the boundary. We therefore prefer interest points with sharp corners to reduce the likelihood that a false interest point is present. Distortion effects are discussed in greater detail section 2.2.4.

In summary, we want to choose features with large basis vectors, sharp angles, and, preferably, no concave angles. To select a query feature, the length of each line segment in the feature is normalized by dividing by the length of the longest segment, and each angle is normalized by dividing by the size of the largest angle. We then define a measure of the quality of the feature: $R_{i}=L_{i} / A_{i}$, where $L_{i}$ is the average normalized segment length for feature $i$, and $A_{i}$ is the average normalized angle for feature $i$. Our initial query feature will be $i$ where $R_{i}$ is maximized over all $i$. The selected feature should be the one least likely to be involved in an overlap of components or objects or to contain a false interest point. Its encoding should also be least sensitive to noise in the input image. If the selected query feature fails, the next best feature is used for searching the feature index.

\subsubsection{Index Search and Hypothesis Generation}

For a given query feature, the index is searched to produce a set of hypotheses, that is, a set of shapes that have a feature similar in shape to the query feature. The details of the search procedure are of course dependent on the PAM being used, but in general a range search is required. The query may specify a tolerance on the similarity measure, which may be 0 if an exact match is required. Each feature found during the search will have an associated list of objects where the feature occurs. These objects may be either rigid, or components of articulated objects. These objects form the set of hypotheses that are passed on to the verification step.

\subsubsection{Hypothesis verification}

If the set of hypotheses is small, then the user may elect to review the set manually and skip the verification stage. In cases where the hypothesis set is large, hypotheses of rigid objects or components of articulated objects may be verified or rejected by overlaying 
the hypothesized shape (suitably transformed to input query coordinates) over the query shape and analyzing the amount of overlap between the two shapes. The transformation parameters are computed by minimizing the distance between the query interest points and the hypothesis interest points in the matched features. This is a non-linear optimization problem, which in general form is a complex and time consuming problem. However, we have a good initial approximation to the minimum by computing the transformation parameters necessary to exactly overlay the basis vectors from the matched features. In addition, the dimensionality of the problem is only 4 (rotation, scale, and translation in $\mathrm{x}$ and $\mathrm{y}$ ) and we only require tolerance to a pixel. Under these conditions it is possible to find a good approximation to the transformation parameters giving minimum distance by using the 'Downhill Simplex method,' (Press, 1988)

If the query shape is from an image that contains overlapping or touching parts, then the shapes may not have similar boundaries, but the hypothesized shape should be contained within the boundary of the query shape. A database shape whose boundary overlap with the query shape exceed a user specified threshold is added to the selection set. At this point, the user may intervene in the selection process to eliminate hypotheses from the selection set that are judged to be poor. If a hypothesis is validated by the user, then interest points in the input image that correspond to that shape are removed from consideration and the process begins again using the remaining interest points to generate a new query feature in an attempt to locate other shapes in the input image. This process continues until there are no longer sufficient interest points to make a valid feature or the user elects to terminate the process.

If multiple components of an articulated object are hypothesized, then the relative orientation of the components can give strong evidence of a match by determining if the articulation points of the components are sufficiently close to one another. The transformation parameters that were calculated to minimize the distance between feature interest points are applied to the articulation point of each respective component. If the components are truly present in the query image, then the transformed articulation point of one component should lie very close to the transformed articulation point of the other component in the query coordinate system. Components that agree, within some tolerance, on the location of their common articulation point, are assumed to be connected about the articulation point, and the articulated object is added to the selection set. It should also be noted that multiple components of one articulated object that are present in a query image should have little difference in the scaling factor needed to map them into the image coordinate system. It is also possible to determine the relative orientation of the components of the articulated object by comparing the rotation parameters of each component.

\subsubsection{Handling distortion effects}

Distortion in a query image may manifest itself in three ways. Interest points may be displaced from their ideal positions, false interest points may be introduced, and legitimate interest points could vanish. The problem of displaced interest points is addressed directly 


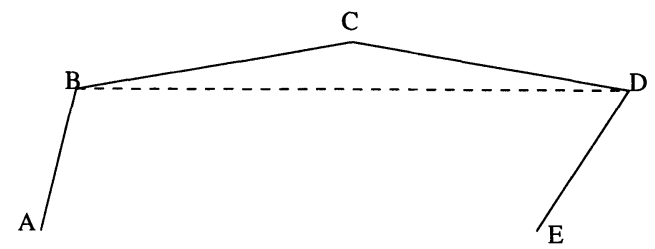

Figure 4: Removal of a feature point determined to be distortion sensitive

by our encoding mechanism and search procedure. Features are encoded as n-dimensional vectors, and a displaced interest point will have a proportional displacement effect on the encoded feature vector. The search process looks for feature vectors within a user-specified range of the query feature vector, so displaced interest points are not likely to cause a failure of the search criteria unless the displacement is very large. They will cause a reduction in the confidence level of the match, but the search tolerance can be increased at will to account for any amount of interest point displacement.

The problem of false interest points is addressed by the query feature selection process. A false interest point is likely to form a shallow angle with its adjacent interest points. The query feature selection algorithm explicitly attempts to avoid shallow angles for exactly this reason.

Missing interest points require a different approach entirely. There is no way to determine where an interest point should have been by examining the input query image only. We address this problem by doing some additional work on the shape boundaries as they are being added to the database. In addition to encoding the features found in the shape boundaries, the angles formed by each interest point are examined, and if sufficiently shallow they are flagged as likely to be missed during query shape analysis if present in an input image. In other words, interest points that are most likely to vanish during query shape processing are identified in the database construction phase. These interest points are then removed from the boundary and the resulting shape is encoded and entered into the index. This is illustrated in figure 2. This is done in addition to the normal case of considering all interest points.

\section{PROTOTYPE SYSTEM}

A prototype system has been implemented to test the performance of the proposed approach. A database consisting of 101 rigid shapes and 6 articulated shapes, with 2 components each was used for experimental purposes. A KD-Btree (Robinson, 1981) is used as the index structure. The KD-Btree is one of the earliest and most primitive point access methods, so we expect that more sophisticated PAMs (Buddy Trees (Seeger, 1987), Grid Files (Nievergelt (1984), etc.) would yield better performance. Indices were created using 


\begin{tabular}{l|c|c|c}
4 Dimensional Index (2210 features) \\
Tolerance & 0.15 & 0.30 & 0.45 \\
\hline \hline Total Seeks & 4292 & 7985 & 12240 \\
Average seeks per search & 37.54 & 70.66 & 108.32 \\
Average hyp. per search & 3.72 & 14.72 & 33.01
\end{tabular}

\begin{tabular}{l|c|c|c}
6 Dimensional Index $(2316$ features $)$ \\
Tolerance & 0.15 & 0.30 & 0.45 \\
\hline \hline Total Seeks & 4004 & 7850 & 12154 \\
Average seeks per search & 35.43 & 69.47 & 107.56 \\
Average hyps. per search & 1.43 & 4.19 & 9.49
\end{tabular}

\begin{tabular}{l|c|c|c}
\multicolumn{4}{c}{ 8 Dimensional Index $(2421$ features $)$} \\
Tolerance & 0.15 & 0.30 & 0.45 \\
\hline \hline Total Seeks & 4441 & 8807 & 13625 \\
Average seeks per search & 39.30 & 77.94 & 120.58 \\
Average hyps. per search & 1.13 & 1.88 & 3.94
\end{tabular}

Table 1: Search Results

4, 5, and 6 interest points per feature. The three indices contained, respectively, 2210, 2316 , and 2421 features (including distorted features).

A performance experiment was conducted by applying the query feature selection algorithm to each of the original shapes present in the database. This test was performed on each of the three indices and at three levels of tolerance in the range search. The results are summarized in table 1. It is noted that the dimensionality had little effect on the number of disk seeks. The higher dimensional cases (more interest points per feature) produced fewer false hypothesis. This is unsurprising as a greater portion of the object is encoded in each feature. Increasing the search tolerance increases the number of false hypothesis and number of seeks, but high tolerance may be required if query images are highly distorted. The tolerance of 0.45 on the 4-dimensional case is clearly too high, however, as almost one third of the shapes in the database are hypothesized on the average.

The prototype system also has a GUI interface written using the XWindow system. Screen shots from this interface illustrate the matching process. A few sample query results are presented in figures $5,6,7$, and 8 . In figure 5, a scissors shape is supplied as an input query (displayed on the left hand side of the figure). Note that this is an articulated object, but the input query contains no information about individual components or articulation point. A feature is selected from the handle (bold lines in figure) and a search of the database produces the correct component (displayed on right hand side of the figure). Actually, in this case, 2 hypotheses were found during the index search. That 


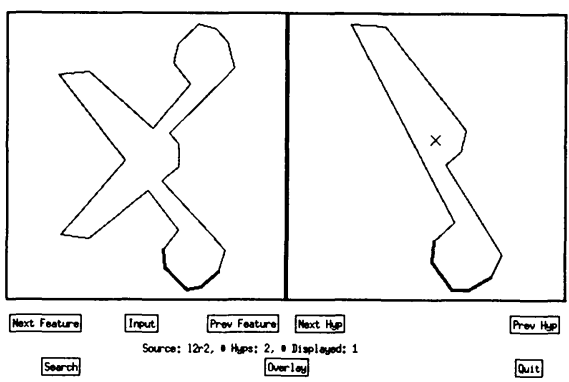

Figure 5: Good Feature Match

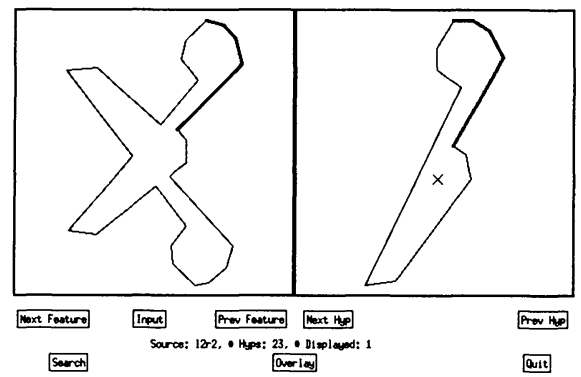

Figure 6: Second Component Match

is, the database contains two features that are within the user's tolerance level of similarity. One hypothesis is displayed with the similar feature highlighted and the user may view other hypothesized shapes by selecting the 'Next Hyp' and 'Prev Hyp' button. Note the ' $\mathrm{X}$ ' that marks the position of the articulation point in the hypothesis. Subsequently the interest points associated with the matched component would be removed from consideration and a feature is selected from the interest points remaining in the query shape. Another search of the index would find the other component (figure 6) and the system finds that they agree on the location of the point of articulation to within a few pixels.

To illustrate the problem of bad query feature choice, examine figure 7 . The input shape is similar to the shapes from figure 5 and 6 , but the scissors are in a more open postion. Here a feature has been chosen with interest points that are from different components of the articulated object. A match is found in the index for that feature. The matched feature has a fairly high error value, indicating that it is not very similar to the query feature. A lower setting on the tolerance level would prevent this specific hypothesis from being generated, but the possibility of matches like this remain. Verification processes, either automated or user-assisted, would eliminated this hypothesis from consideration. 


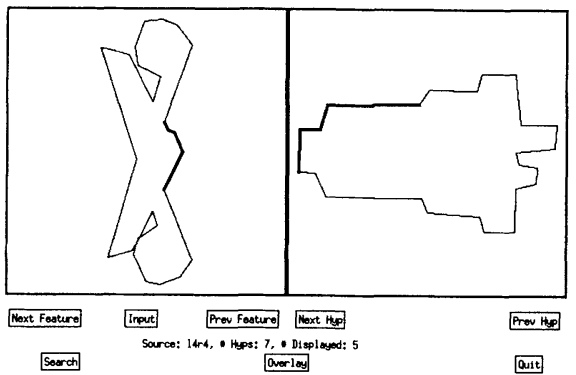

Figure 7: Bad Feature Selection

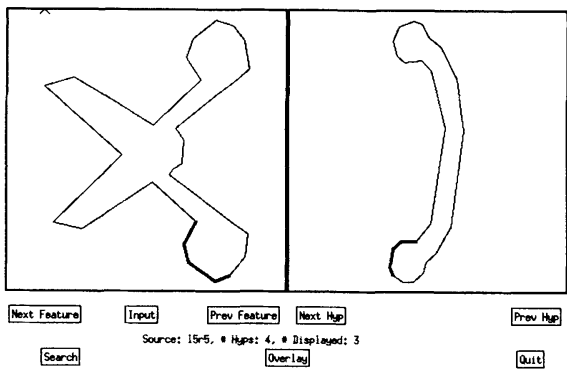

Figure 8: Good Feature, Bad Match 


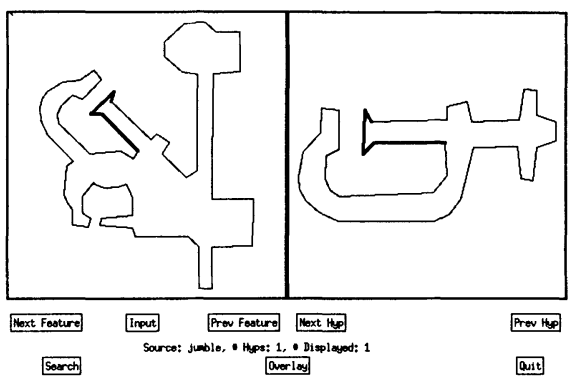

Figure 9:

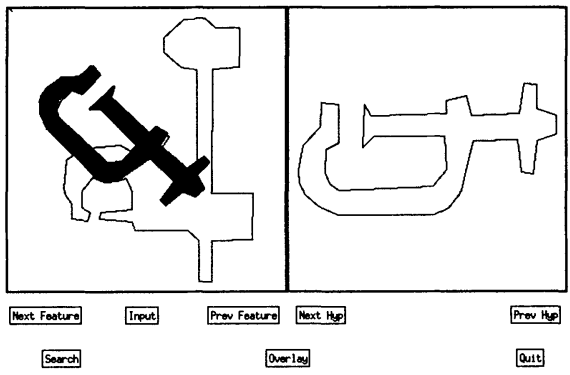

Figure 10:

It is of course possible to find similar features even without having an unacceptable query feature choice. Figure 8 shows a match for a feature, but the shape it matches is not globally similar in shape to the scissors. In general it is not possible to avoid these cases altogether, but the verification stage can automatically reject many bad hypotheses and the user can manually decide to reject others.

To illistrate some additional features of the prototype system, consider figure 9. Here the query image consists of several objects from our database that are overlapping. The feature chosen by the program has been rejected by the user who may interactively specify a query feature by selecting the "Next Feature" and "Prev Feature" buttons. The user then selected the "Search" button to generate hypotheses. A single hypothesis was found and is shown on the right hand side of the window. In figure 10, the user has selected the "Overlay" button to see how well the hypothesis matches the query after suitable translation, rotation, and scaling.

Figure 11 shows a feature choice that generates many hypotheses (the error tolerance was set pretty high in testing). The first hypothesis seems to be reasonable. In figure 12, the user has selected the "Next Hyp" button to display an alternative hypothesis. 


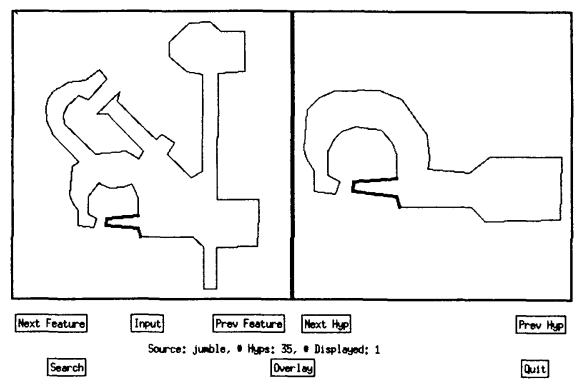

Figure 11:

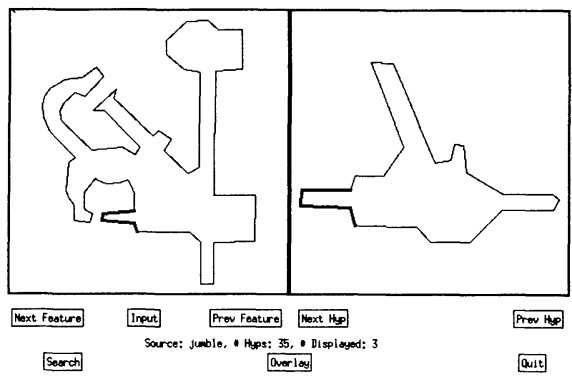

Figure 12:

If desired, the "Prev Hyp" button allows the user to move backward through the list of hypotheses. The hypothesis list is sorted in increasing order of error, so the best one appears first. The poor hypothesis is overlaid on the query shape in figure 13 .

The prototype also allows the user to interactively sketch a shape to be used as a query. In figure 14, a shape was sketched with the intention of being similar to the hypothesis of figures 12 and 13. Notice that a match did occur and the overlay illustrated in figure 15 shows a high degree of global similarity.

\section{CONCLUSIONS}

Existing techniques for shape similarity based retrieval in image databases are limited to rigid shapes. Our proposed iconic index structure is general enough to handle articulated shapes as well as rigid shapes and flexible enough to handle retrieval of isolated shapes as well as overlapping or touching shapes. A prototype system presented and performance analysis reveal it to be efficient and effective. We are continuing to evaluate performance of various point access methods with different sized image databases and other shape representations and similarity measures. We are also investigating the extension of the 


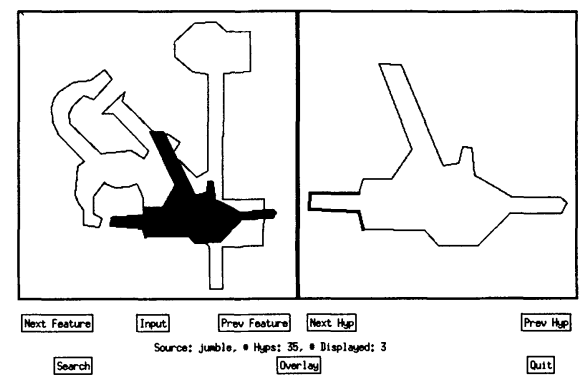

Figure 13:

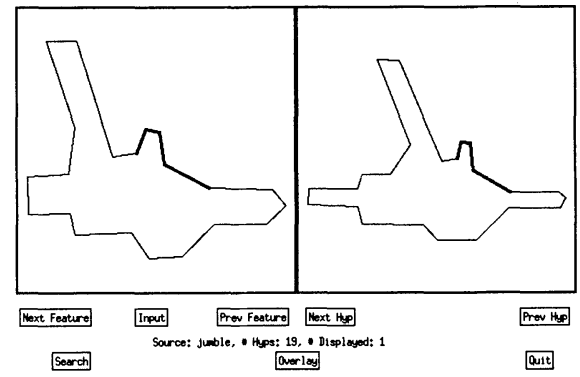

Figure 14:

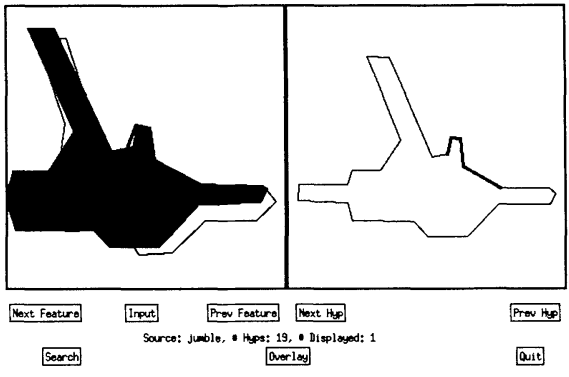

Figure 15: 
technique to 3 dimensional shapes.

\section{ACKNOWLEDGMENTS}

This project was supported in part by NASA-Langley Research Center Grant NAG-11276, by the Biomedical Research Support Grant BRSG SO7 RRO7114-21 of the National Institute of Health

\section{REFERENCES}

Chang, S. K. and Yan, C. W.(1987) Iconic Indexing of 2D Strings. IEEE Transactions on Pattern Analysis and Machine Intelligence, 9(3), 413-428

Barber, R. , Equitz, W. , Faloutsos, C. , Flickner, M. , Niblack, W. , Petkovic, D. , and Yanker, P. (1993) Query by Content for Large On-Line Image Collections. Research Report RJ 9408 (82660), IBM Research Division.

Beinglass, A. and Wolfson, H. (1991) Articulated Object Recognition, or: How to Generalize the Generalized Hough Transform, Proceedings of the 1991 IEEE Computer Society Conference on Computer Vision and Pattern Recognition,, Lhaina, Maui, Hawaii, 461-465.

Faloutsos, C. , Flickner, M. , Niblack, W. , Petkovic, D. , Equitz, W. , and Barber, R. (1993) Efficient and Effective Querying by Image Content. Research Report RJ 9453 (83074), IBM Research Division.

Gary, J. E . and Mehrotra, R. (1993) Shape Similarity-Based Retrieval Using a Structural Feature Index. Information Systems, 18(7), 525-537.

Grosky, W. I. and Mehrotra, R. (1990) Index-Based Object Recognition in Pictorial Data Management. Computer Vision, Graphics, and Image Processing 52(3), 416-436.

Grosky, W. I. , Neo, P. , and Mehrotra, R. (1989) An Iconic Index for Model-Based Matching. Proceedings of the Fifth International Conference on Data Engineering, Los Angeles, California, 180-187.

Grosky, W. I. , Neo, P. , and Mehrotra, R. (1992) A Pictorial Index Mechanism for Model-Based Matching. Data and Knowledge Engineering, 8, 309-327.

Jagadish, H. V. (1991) A Retrieval Technique for Similar Shapes. Proceedings of the ACM SIGMOD International Conference on the Management of Data, Denver Colorado, 208-217.

Lowe, D. G. (1991) Fitting Parameterized Three-Dimensional Models to Image. IEEE Transactions on Pattern Analysis and Machine Intelligence, 13(5), 441-450.

Mehrotra R. and Gary J. E. (1993), Feature-Based Retrieval of Similar Shapes. Proceeding of the Nineth International Conference on Data Engineering, Vienna, Austria, 108-115. 
Nievergelt, J. , Hinterberger, H. , and Sevcik, K. C .(1984) The Grid File: An Adaptable Symmetric Multikey File Structure. ACM Transactions on Database Systems, 9(1), $38-71$.

Press, William H. (1988) Numerical Recipes in C. Cambridge University Press.

Robinson, J. T. (1981) K-D-B-tree: A Search Structure for Large Multidimensional Dynamic Indices. Proc. ACM SIGMOD Conference on the Management of Data, Ann Arbor, Michigan, 10-18.

Seeger, B. and Kriegel, H. P. (1987) The Buddy Tree: An Efficient and Robust Access Method for Spatial Database Systems. Proc. 16th International Conference on Very Large Databases, Brighton, U.K., 590-601.

Tsang, W. M. , Yuen, P .C. , and Lam, F. K. (1994) Detection of Dominant Points on an Object Boundary: a Discontinuity Approach. Image and Vision Computing, 12(9), $547-557$.

Wuescher, D. M. and Boyer, K. L. (1991) Robust Contour Decompositions using a Constant Curvature Criterion. IEEE Transactions on Pattern Analysis and Machine Intelligence, 13(1), 41-51.

\section{BIOGRAPHY}

Rajiv Mehrotra received a B. Tech degree in Electrical Engineering from Kanpur University, India, in 1977, an M. Tech degree in Electrical Engineering from the Indian Institute of Technology, Kanpur, India, in 1979, an M. A. and a Ph. D. in Computer Science from Wayne State University, Detroit, Michigan, in 1983 and 1986, respectively.

Dr. Mehrotra is currently an Associate Professor in the Department of Mathematics and Computer Science of the University of Missouri-St. Louis. His prior employments include faculty positions at University of Kentucky, Lexington, KY (Associate Professor of Computer Science and Electrical Engineering, 1990-94) and University of South Florida, Tampa, Florida (Assistant Profsssor of Computer Science and Computer Engineering(1986-90).

His current research interests include multimedia information management systems and visual information procesing and management. He was a co-guest editor (with B. P. Berra, F. Golshani, and O. Sheng) of a special section of IEEE Transactions on Knowledge and Data Engineering on Multimedia Information Systems (August 1993). He coedited (with M. R. Varanasi) a reprint collection entitled "Multirobot Systems," published by the IEEE Computer Society Press. He was a co-guest editor (with W. I. Grosky) of a special issue of IEEE Computer on Image Database Management (December 1989).

Dr. Mehrotra is a senior member of IEEE and a member of ACM.

James Gary received a B. A. in Physics and Mathematics from Indiana University, Bloomington, Indiana in 1982 and an M. S. in Computer Studies from North Carolina 
State University, Raleigh, North Carolina in 1985. He is currently pursuing a $\mathrm{Ph}$. D. in Computer Science at the University of Kentucky, Lexington, Kentucky.

Mr. Gary has worked as an instructor at Winston Salem State University in North Carolina and at the University of Kentucky. He will be joining the Mathematical Sciences faculty at Cameron University in Lawton, Oklahoma in the Fall of 1995. His research interests include Image Databases, Computer Graphics, Image Processing, Programming Languages, and Computer Vision. 